\title{
INVERSE SCATTERING FOR A DIELECTRIC CYLINDER
}

\author{
Huang-Tien Lin and Yean-Woei Kiang* \\ Department of Electrical Engineering, National Taiwan University \\ Taipei, Taiwan, Republic of China \\ Tel: 886-2-3635251, Fax: 886-2-3671909
}

\begin{abstract}
The problem of reconstructing both the shape and the relative permittivity of a homogeneous dielectric cylinder in free space from the measurement of scattered field around a circle is investigated. In simulation, the Newton-Kantorovitch algorithm, moment method and singular value decomposition (S.V.D.) technique are used to solve a set of nonlinear integral equations. Numerical results show that, with multiple incident directions, good reconstruction is obtained. This algorithm can be applied at a single frequency without limitation on the permittivity value.
\end{abstract}

\section{Introduction}

The inverse scattering problem is to recover information about some inaccessible region from the scattered wave fields measured outside. This problem has attracted increasing attention owing to its important applications, such as remote sensing, medical imaging, nondestructive evaluation, etc. Exact inverse methods have been developed for the problem where the parameters of interest depend only on a single spatial dimension, and accurate numerical algorithms are also available. However, it is much more difficult to solve the problem if the properties of the scatterer depend on more than one spatial dimension.

In the past few years, two kinds of approaches have been developed for the inverse scattering of dielectric objects. The first is an approximate approach, such as the Born approximation. The second is to solve relevant exact equations by numerical methods. The latter needs no approximation in formulation, but its computation is more complex than the former.

In this paper, the inverse scattering of a homogeneous dielectric cylinder in free space is numerically investigated. In section II, the theoretical formulation for this problem is briefly described. Numerical results are shown in section III. Finally, some conclusions are drawn.

\section{Formulation}

Let us consider a dielectric cylinder in free space with permittivity $\epsilon_{\mathrm{r}} \epsilon_{0}$ and permeability $\mu_{0}$ as shown in Fig.1. The axis of this cylinder is parallel to the zaxis with the displaced position at $\left(X_{0}, Y_{0}\right)$. Its cross section can be described in polar coordinates with respect to $\left(X_{o}, Y_{o}\right)$ by the equation $\rho=F(\theta)$, i.e., the object is of starlike shape.

The incident wave is a time-harmonic plane wave with electric field vector parallel to the $z$-axis (TM polarization), i.e.,

$$
\vec{E}_{\mathrm{i}}(x, y)=E_{\mathrm{o}} e^{-j k(x \sin \phi-y c o s \phi)} \hat{z}
$$




$$
\vec{H}_{i}(x, y)=-\frac{E_{o}}{\eta}(\cos \phi \hat{x}+\sin \phi \hat{y}) e^{-j k(x \sin \phi-y \cos \phi)}
$$

where $\phi$ is the incident angle, and $\eta$ is the intrinsic impedance of free space. The scattered field $\vec{E}$, can be expressed by the following integral

$$
\vec{E}_{s}(\vec{r})=\int_{0}^{2 \pi}\left[j \omega \mu_{0} G_{0}\left(k\left|\vec{r}-\vec{r}^{\prime}\right|\right) \vec{J}\left(\theta^{\prime}\right)+\vec{M}\left(\theta^{\prime}\right) \times \nabla^{\prime} G_{0}\left(k\left|\vec{r}-\vec{r}^{\prime}\right|\right)\right] d \theta^{\prime}
$$

where $\vec{J}$ and $\vec{M}$ are respectively proportional to the equivalent surface electric and magnetic current densities with proportion constant being the Jacobian of the shape function, and $G_{0}$ and $G_{2}$ are the Green's functions in free space and in a homogeneous space with relative permittivity $\epsilon_{r}$ respectively.

From the boundary conditions that the tangential electric and magnetic fields should be continuous across the surface of the object, we have the following two integral equations[1]

$$
\begin{aligned}
\hat{n} \times \vec{E}_{i}(\vec{r})= & -\hat{n} \times \int_{0}^{2 \pi}\left[j \omega \mu_{0}\left(G_{2}+G_{0}\right) \vec{J}\left(\theta^{\prime}\right)+\vec{M}\left(\theta^{\prime}\right) \times \nabla^{\prime}\left(G_{2}+G_{0}\right)\right] d \theta^{\prime} \\
\hat{n} \times \vec{H}_{i}(\vec{r})= & -\hat{n} \times \int_{0}^{2 \pi}\left[j \omega\left(\epsilon_{2} G_{2}+\epsilon_{0} G_{0}\right) \vec{M}\left(\theta^{\prime}\right)-\vec{J}\left(\theta^{\prime}\right) \times \nabla^{\prime}\left(G_{2}+G_{0}\right)\right. \\
& \left.-\frac{\rho_{m}\left(\theta^{\prime}\right)}{\mu_{0}} \nabla^{\prime}\left(G_{2}+G_{0}\right)\right] d \theta^{\prime}
\end{aligned}
$$

where $\rho_{m}$ is proportional to the equivalent surface magnetic charge density and $\hat{n}$ is the outward unit normal on the object surface.

For the direct problem, the scattered field $\vec{E}$, is to be calculated assuming that the shape and the relative permittivity of the object are known. We may solve $\vec{J}$ and $\vec{M}$ from Eqs.(4) and (5) by the moment method and then calculate $\vec{E}$, from Eq.(3), which can be used as the measured data in simulating the inverse problem.

The inverse problem to be considered is: given the scattered field $\vec{E}_{s}$ measured outside on a circle of radius $a$, determine the shape and the relative permittivity of the object.

Assume that the shape function of the object can be expanded as

$$
F(\theta) \simeq \sum_{n=0}^{N / 2} A_{n} \cos (n \theta)+\sum_{n=1}^{N / 2} A_{n}^{\prime} \sin (n \theta)
$$

where $A_{n}$ and $A_{n}^{\prime}$ are real numbers. These coefficients, together with the relative permittivity $\epsilon_{r}$ and the relative center $\left(X_{o}, Y_{o}\right)$ of the object, are to be reconstructed.

By applying the Newton-Kantorovitch algorithm[2], Eqs.(3),(4), and (5) are cast into a set of linear equations. Then the incremental coefficients $\delta A_{n}$, 
$\delta A_{n}^{\prime}, \delta X_{o}, \delta Y_{o}$, and $\delta \epsilon_{r}$ are solved by the moment method[3] and the S.V.D. technique[4], so that $A_{n}, A_{n}^{\prime}, X_{o}, Y_{o}$, and $\epsilon_{r}$ can be corrected at each iteration. When the field error is less than some prescribed value, the iterative procedures will be stopped and the reconstructed results are then obtained.

\section{Numerical Results}

Let the incident plane wave be of unit amplitude and the free-space wavelength $\lambda_{o}$ is $0.1 \mathrm{~m}$ (i.e., $f=3 G H z$ ). For simulation, the object is illuminated by the incident plane wave from three directions and 14 measurement points at equal spacing are used along a circle of radius $a=\lambda_{0}$. In applying the moment method, the boundary of the object is divided into segments and pulse basis functions are used for solving $\vec{J}$ and $\vec{M}$. The number of basis functions for the direct problem is about two times of that for the inverse problem, since it is crucial that the synthetic data generated through a forward solver are different from those obtained by the inverse solver.

In case 1(Fig.(2)), the exact shape function $F(\theta)=[0.012+0.0015 \cos (2 \theta)+$ $0.0013 \cos (3 \theta)] \mathrm{m}$ and $\epsilon_{r}=2.1$. After 10 iterations, the result shows that the rms (root mean square) error of the shape is about $3 \%$ and the reconstructed $\epsilon_{\mathrm{r}}=2.147$. In case 2(Fig. (3)), the exact shape function $F(\theta)=[0.018-$ $0.003 \cos (3 \theta)] \mathrm{m}$ and $\epsilon_{\mathrm{r}}=2.4$. After 10 iterations, the result shows that the rms error of the shape is about $4 \%$ and the reconstructed $\epsilon_{r}=2.417$.

During iteration, the relative permittivity converges first. Then the S.V.D. technique is used to obtain a better reconstructed shape. The reconstructed results are in good agreement with the exact values.

\section{Conclusions}

An efficient iterative algorithm for reconstructing both the shape and the relative permittivity of a homogeneous dielectric cylinder has been proposed. It is based on the Newton-Kantorovitch algorithm, moment method, and S.V.D. technique to solve the nonlinear equations. In simulation, we use three incident directions and only one frequency. The shape and the permittivity of the object can be successfully reconstructed even when the permittivity is fairly large and the Born approximation is no longer valid.

\section{References}

[1] K. M. Chen, "A mathernatical formulation of the equivalence principle," IEEE Trans. Microwave Theory Tech., vol.37, pp.1576-1581, October. 1989.

[2] A. Roger, "Newton-Kantorovitch algorithm applied to an electromagnetic inverse problem," IEEE Trans. Antennas Propagat. , vol.AP-29, pp. 232238, Mar. 1981.

[3] R. F. Harrington, Field Computation by Moment Methods, Macmillan, New York, 1968.

[4] W. H. Press, B. P. Flannery, S. A. Teukolsky, and W. T. Vetterling, Numerical Recipes: The Art of Scientific Computing, Cambridge, UK: Cambridge University, 1986. 


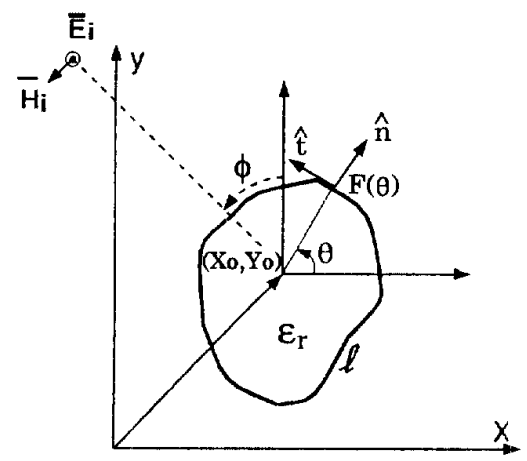

Fig.1. Geometry of the problem in $(x, y)$ plane.
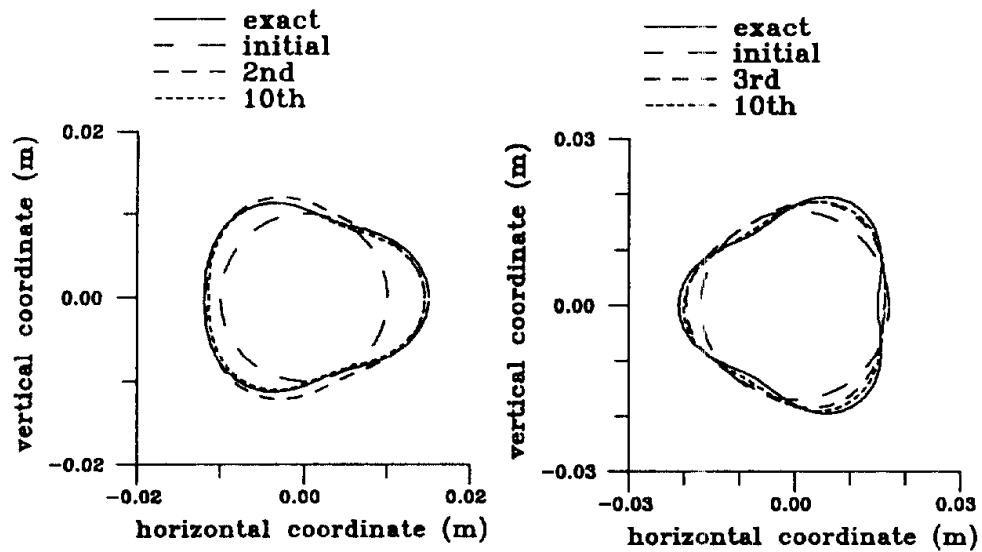

Fig.2. Reconstructed results for the object in case 1 . The dashed curves are calculated shapes in iteration process, and the rms error is $3 \%$ after 10 iterations. The exact relative permittivity $\epsilon_{r}=2.1$, and the reconstructed value is 2.147 .

Fig.3. Reconstructed results for the object in case 2 . The dashed curves are calculated shapes in iteration process, and the rms error is $4 \%$ after 10 iterations. The exact relative permittivity $\epsilon_{r}=2.4$, while the reconstructed value is 2.417 . 